\title{
\begin{tabular}{l|l} 
Mibraries & DSpace@MIT
\end{tabular}
}

\author{
MIT Open Access Articles
}

\section{Thermoelectrically Pumped Light-Emitting Diodes Operating above Unity Efficiency}

The MIT Faculty has made this article openly available. Please share how this access benefits you. Your story matters.

Citation: Santhanam, Parthiban, Dodd Gray, and Rajeev Ram. “Thermoelectrically Pumped Light-Emitting Diodes Operating Above Unity Efficiency." Physical Review Letters 108.9 (2012). (C) 2012 American Physical Society

As Published: http://dx.doi.org/10.1103/PhysRevLett.108.097403

Publisher: American Physical Society

Persistent URL: http://hdl.handle.net/1721.1/71563

Version: Final published version: final published article, as it appeared in a journal, conference proceedings, or other formally published context

Terms of Use: Article is made available in accordance with the publisher's policy and may be subject to US copyright law. Please refer to the publisher's site for terms of use. 


\title{
Thermoelectrically Pumped Light-Emitting Diodes Operating above Unity Efficiency
}

\author{
Parthiban Santhanam, Dodd Joseph Gray, Jr., and Rajeev J. Ram* \\ Research Lab of Electronics, Massachusetts Institute of Technology, Cambridge, Massachusetts 02139, USA
}

(Received 9 August 2011; revised manuscript received 24 October 2011; published 27 February 2012)

\begin{abstract}
A heated semiconductor light-emitting diode at low forward bias voltage $V<k_{B} T / q$ is shown to use electrical work to pump heat from the lattice to the photon field. Here the rates of both radiative and nonradiative recombination have contributions at linear order in $V$. As a result the device's wall-plug (i.e., power conversion) efficiency is inversely proportional to its output power and diverges as $V$ approaches zero. Experiments directly confirm for the first time that this behavior continues beyond the conventional limit of unity electrical-to-optical power conversion efficiency.
\end{abstract}

The presence of entropy in incoherent electromagnetic radiation permits semiconductor light-emitting diodes (LEDs) to emit more optical power than they consume in electrical power, with the remainder drawn from lattice heat $[1,2]$. Here we report the first experimental observation of electroluminescence in which the ratio of detected optical power to supplied electrical power, known commonly as the wall-plug efficiency $\eta$ (or as the heating coefficient of performance), exceeds unity. Although this result is of basic scientific interest, recently it has also drawn interest in the applied community for its potential in lighting and solid-state cooling [3-10].

In an electroluminescently cooled LED, electrons and holes are first excited by the applied voltage. The total amount of electrical work supplied per excitation is the product of the electron's charge $q$ and the bias voltage $V$, and when $q V$ is zero the device is in thermodynamic equilibrium. Upon excitation, some of the electrons and holes, a fraction defined as the external quantum efficiency $\eta_{\mathrm{EQE}}$, relax by radiative recombination and generate photons that exit the device. If each injected electron-hole pair emits a photon of energy $\hbar \omega$ with a probability $\eta_{\mathrm{EQE}}$ but requires just $q V$ in work for excitation, we may write the following expression for the wall-plug efficiency $\eta[1,3]$ :

$$
\eta=\frac{\hbar \omega}{q V} \eta_{\mathrm{EQE}} .
$$

The observation of light emission with photon energy $\hbar \omega$ in excess of the electrical input energy per electron $q V$ is readily accessible in LEDs at a variety of wavelengths. At these operating points, the electron population is pumped by a combination of electrical work and Peltier heat originating in the semiconductor's lattice; this thermoelectric heat exchange is nonuniformly distributed throughout the device [5,11]. As early as 1953 this phenomenon had been experimentally observed in a $\mathrm{SiC}$ emitter and connected physically to the Peltier effect [12]. Nevertheless, net cooling, or equivalently electroluminescence with wallplug efficiency greater than unity, has eluded direct observation for more than five decades.
Early measurements [12-14] of light emission from semiconductor diodes were followed closely by theoretical developments. Beginning with Tauc in 1957, a body of literature theoretically establishing the basic thermodynamic consistency of electroluminescent cooling and exploring its limits began to emerge [1,2,15-17]. In 1964, Dousmanis et al. experimentally demonstrated that a GaAs diode could produce electroluminescence with an average photon energy 3\% greater than $q V$ [18]. Still, net cooling was not achieved due to competing nonradiative recombination processes [19]. The authors concluded that a high value of $\eta_{\mathrm{EQE}}$ was a requirement for any direct experimental observation.

In the past decade, several modeling and design efforts have aimed to raise $\eta_{\mathrm{EQE}}$ toward unity by maximizing the fraction of recombination that is radiative $[3,4,7]$ and employing photon recycling to improve photon extraction $[3,4,6]$. Attempts to experimentally observe electroluminescent cooling with $\eta$ near $100 \%$ have been made [9], and some continue to be active [10]. These attempts have focused exclusively on the regime in which $q V$ is on the order (i.e., at least 50\%) of the material band gap $E_{g} \approx \hbar \omega$. As $q V$ is lowered well below $E_{g}$, the electron and hole populations decrease exponentially following a Boltzmann distribution with decreasing chemical potential. Since an excited electron in a direct band gap semiconductor may relax either by recombining with a hole and emitting a photon or alternatively by scattering into a state associated with a lattice imperfection and emitting phonons, small forward bias voltages $q V \ll E_{g}$ are typically precluded by the requirement of high $\eta_{\mathrm{EQE}}$. Moreover, recent theoretical work by Heikkila, et al. [3] found that for a conventional LED with an undoped active region, $\eta_{\mathrm{EQE}}$ should approach zero at low bias, and effectively dismissed the low-bias regime for conventional LEDs. This conclusion was reached by assuming that the active region carrier concentrations $n$ and $p$ would always exceed the intrinsic concentration $n_{i}$ at operating points of interest.

By exploring a regime where this assumption is no longer valid, we have observed net electroluminescent 
cooling in a LED with $\eta_{\mathrm{EQE}} \approx 3 \times 10^{-4}$. Despite this low $\eta_{\mathrm{EQE}}$, the collected light power to input electrical power ratio $\eta$ exceeded $200 \%$, as seen in Fig. 1(a).

The LED was operated in a regime that differs from that of recent proposals $[6,7,9]$. This difference is captured by three characteristic energies: the electrical energy $q V$, the thermal energy $k_{B} T$, and the material band gap $E_{\text {gap. }}$. Here cooling was observed by applying a $70 \mu \mathrm{V}$ forward bias, so that $q V$ was several hundred times smaller than $k_{B} T$. At voltages $V<k_{B} T / q$, hereafter referred to as the low-bias regime, $\eta_{\mathrm{EQE}}$ becomes voltage independent and further reductions in voltage increase the wall-plug efficiency $\eta$. By moving to narrow band gap materials and raising the emitter lattice temperature as Berdahl originally suggested [19], the power available in the low-bias regime was increased by several orders of magnitude.
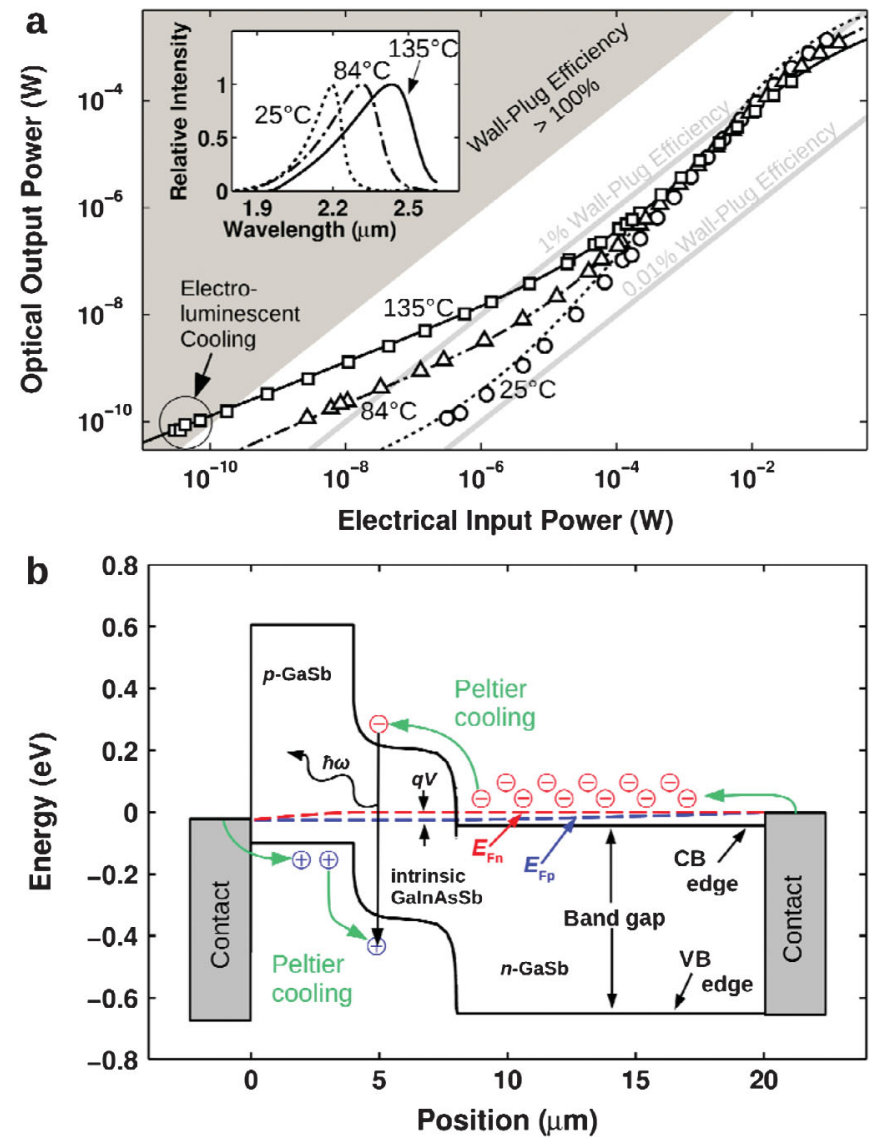

FIG. 1 (color). (a) LED output optical power versus input electrical power. The inset shows the experimental temperature dependence of the emission spectrum for a typical device. The lines denote the results of a numerical model. The discrete markers denote experimental data. (b) Band diagram at $26 \mathrm{mV}$ forward bias. Peltier heat exchange occurs at various positions within the device. When a small forward bias is applied, most of the energy required to inject electrons and holes into the InGaAsSb active region is drawn from the lattice and the device operates as a heat pump. $(\mathrm{CB}$, conduction band; $\mathrm{VB}$, valence band.)
In Fig. 1(a), the output optical power is plotted against the input electrical power for a double heterojunction LED designed to emit around $2.15 \mu \mathrm{m}$ [20]. The band diagram for the LED at low bias is depicted in Fig. 1(b). The applied voltage combines with Peltier heat drawn from the lattice to inject electron-hole pairs into an active region consisting of an alloy of indium, gallium, arsenic, and antimony. The injection process depicted in Fig. 1(b) drives both radiative and nonradiative recombination. At low bias, these processes result in a small but measurable forward current and net outgoing photon flux as shown in Fig. 2.

We have used lock-in photodetection [21] to directly detect the emission of $69 \pm 11 \mathrm{pW}$ of optical power from an existing $\operatorname{In}_{0.15} \mathrm{Ga}_{0.85} \mathrm{As}_{0.13} \mathrm{Sb}_{0.87}$ infrared LED [20] as the result of $29.9 \pm 0.1 \mathrm{pW}$ of input electrical power. This single measurement represents the high-temperature lowpower end point of a larger data set characterizing the supplied current and voltage along with the resulting optical output power as the LED's temperature was varied between $25^{\circ} \mathrm{C}$ and $135^{\circ} \mathrm{C}$. As shown in the inset of Fig. 1(a), at $135^{\circ} \mathrm{C}$ these devices emit a broad light spectrum centered at a wavelength near $2.42 \mu \mathrm{m}$ with a full width at half maximum of $0.29 \mu \mathrm{m}$. Since the photodetector remained unheated $\left(\approx 25^{\circ} \mathrm{C}\right)$, even without bias the LED's $135^{\circ} \mathrm{C}$ active region emits approximately $40 \mathrm{nW}$ of blackbody radiation in this wavelength range; the lock-in technique was necessary to separate the $69 \mathrm{pW}$ of electrically driven optical power from the portion of that blackbody background which was incident on the detector surface.

The high-temperature measurements span roughly 7 orders of magnitude in output power. Below approximately $1 \mu \mathrm{W}$, the optical power was measured using a lock-in technique. The LED was placed electrically in series with an unheated resistor and the combined load was biased

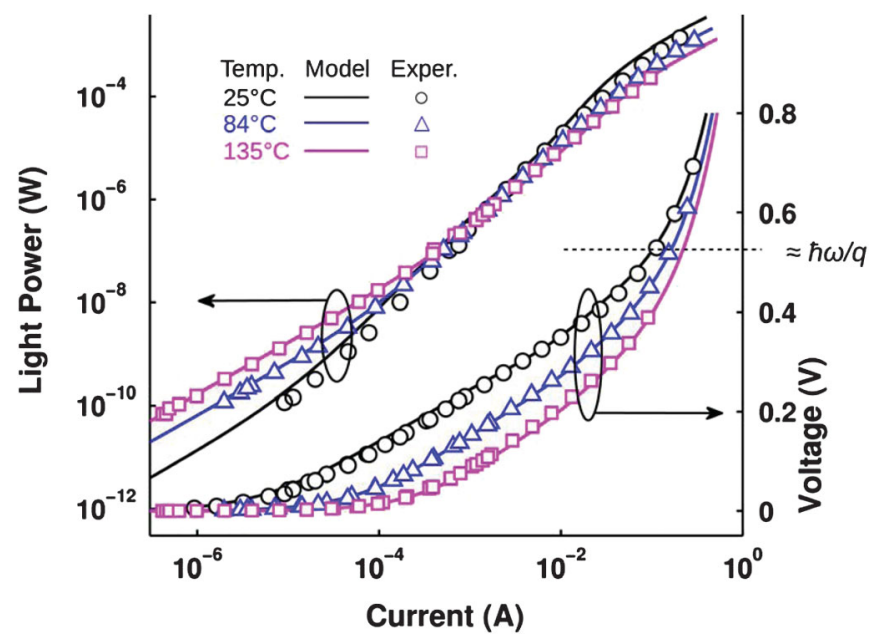

FIG. 2 (color). Light-current and current-voltage characteristics for the LED. Measurable current flows and measurable light is emitted even as $q V$ is reduced well below the average energy $\hbar \omega$ of the emitted photons. 
with a $1 \mathrm{kHz}$ on-off voltage square wave. This resistor dominated the load across the function generator so that the LED was approximately current biased. The optical power was detected by a long-wavelength InGaAs $p-i-n$ photodiode whose photocurrent signal was amplified and measured by a transimpedance amplifier connected to a digital lock-in amplifier. The phase of the optical power signal remained fixed as the excitation voltage was reduced, indicating that even at low power the measured signal was the result of LED electroluminescence [21]. The uncertainties presented result primarily from background electrical noise in the lock-in measurement of optical power. This noise was observed to be zero mean, with no preferential phase relationship to the excitation signal. Above $100 \mathrm{nW}$, an overlapping power measurement was made using a de voltage source and digital multimeter. The lockin optical power measurements were in agreement with the dc to within the experimental uncertainty. Data acquired by both methods in the overlapping power range appear in the figures.

Figure 2 shows the current and optical output power measured at each voltage. Reorganizing this data as shown in Fig. 3(a) reveals that, at currents corresponding to the low-bias regime, $\eta_{\mathrm{EQE}}$ is observed to be independent of current. Voltages $V \ll k_{B} T / q$ constitute a small deviation from thermodynamic equilibrium and drive net photon generation as a linear response in much the same way as a small excess of reactants drives a chemical reaction. Applying a forward bias raises the steady-state concentration of electrons $n_{0}$ and holes $p_{0}$ by $\delta n$ and $\delta p$, respectively. The total radiative recombination rate is proportional to the product $n p$, where $n=n_{0}+\delta n$ and $p=p_{0}+\delta p$. Since recombination balances generation at equilibrium, the cross terms of this product give the net recombination rate to leading order in the deviations $\delta n$ and $\delta p$. Since net light emission is linearly proportional to $\delta n$ and $\delta p$, and $\delta n$ and

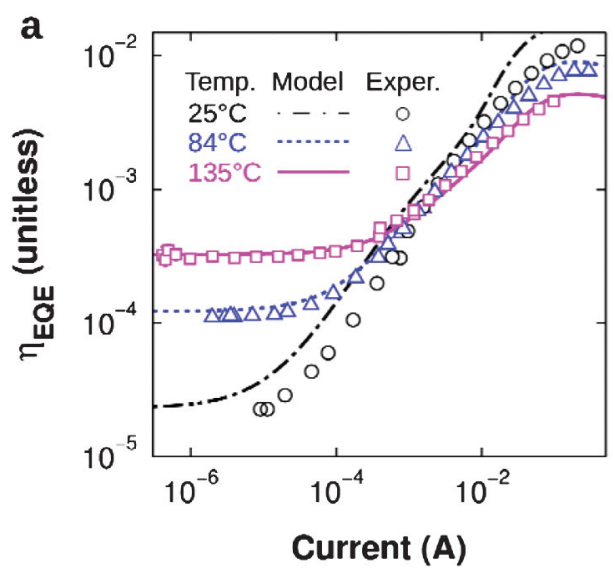

$\delta p$ are linear in $V$, at low bias an LED's quantum efficiency is finite, including as $V$ approaches zero.

In the presence of defects in the semiconductor lattice, $\eta_{\mathrm{EQE}}$ is often small at low bias because nonradiative Shockley-Read-Hall (SRH) recombination associated with defect trap states dominates [22,23]. Trap states compete with equilibrium holes for the capture of excess electrons, and with equilibrium electrons for the capture of excess holes. Since the equilibrium carrier densities rise rapidly with temperature while the trap density does not, low-bias $\eta_{\mathrm{EQE}}$ also rises with temperature. In this experiment a $24 \%$ increase in the absolute temperature resulted in a tenfold rise in low-bias $\eta_{\mathrm{EQE}}$.

Figure 3(b) shows that the wall-plug efficiency varies inversely with optical output power at low bias. This observation agrees with Eq. (1) when $\eta_{\mathrm{EQE}}$ is voltage independent and the $I-V$ characteristic is linear through the origin, as in the low-bias regime.

Figure 3(b) also shows that this behavior continues beyond the point of unity wall-plug efficiency, indicating net electroluminescent cooling. Unlike strategies for cooling which work only above some minimum junction voltage $[4,6,7]$, as shown by Tauc under the assumption of constant $\eta_{\mathrm{EQE}}$ the low-bias strategy theoretically permits unbounded wall-plug efficiency at infinitesimal power [1]. Note that the existence of such a low-bias regime is a general, material-independent physical property of LEDs.

Although the Peltier heat exchange of the injection process is highly nonuniform, on average the device remains very slightly cooled so that in steady state the thermal energy required to pump the emitter flows in from the ambient environment. The net cooling power $P_{\text {cool }}$ is given by the difference between the emitted optical power and the input electrical power and is shown in Fig. 4(a). In terms of the zero-bias resistance $R$ and the current through the device $I$, the net cooling power is given by

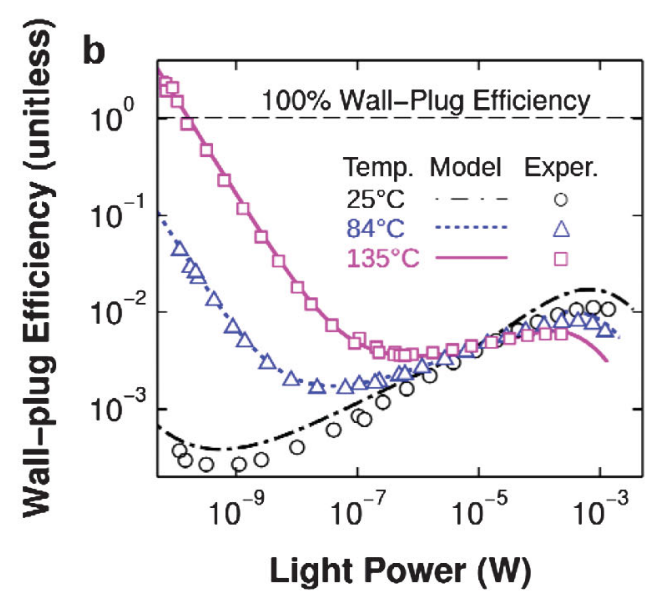

FIG. 3 (color). (a) External quantum efficiency $\eta_{\mathrm{EQE}}$ versus current and (b) wall-plug efficiency $\eta$ versus output optical power. External quantum efficiency $\eta_{\mathrm{EQE}}$ asymptotically approaches a constant value in the low-bias regime (where $V<k_{B} T / q$ ). As a result, in this regime output power is linear in current while input power is quadratic, and $\eta$ is inversely proportional to output power. This behavior continues beyond unity efficiency indicating electroluminescent cooling. 

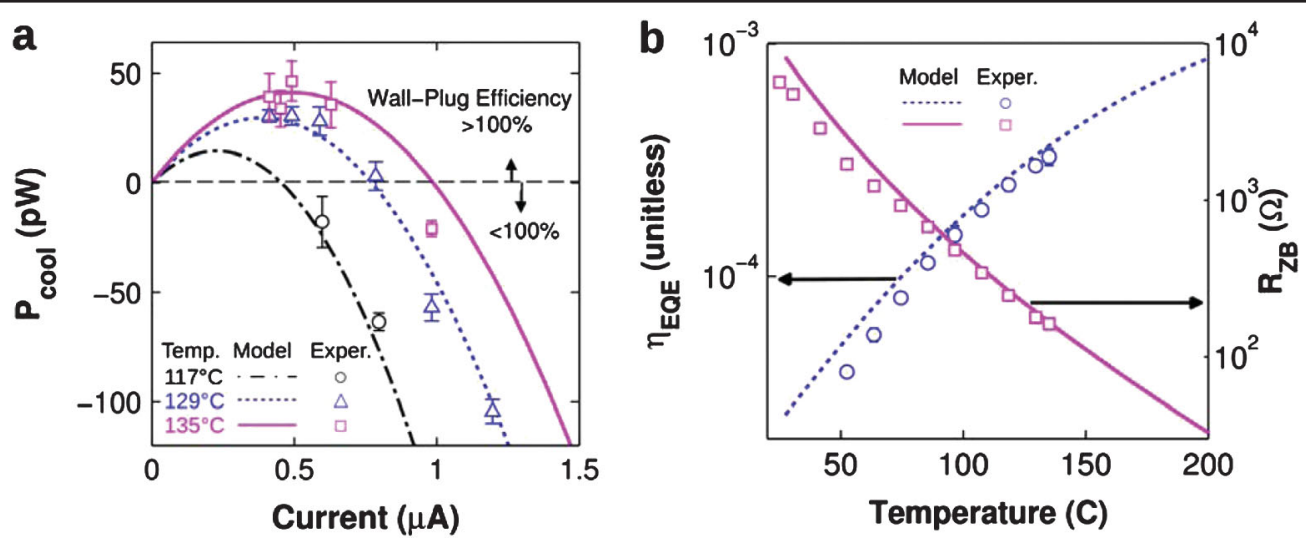

FIG. 4 (color). (a) Cooling power versus current at high temperature and (b) low-bias external quantum efficiency and zero-bias (ZB) resistance versus temperature. The parabolic dependence of cooling power on current is indicative of a linear cooling process competing with a quadratic heating process. Increases in $\eta_{\mathrm{EQE}}$ and reductions in $R$ lead to improved heat pumping power as the temperature rises. Numerical results suggest that this trend should continue to higher temperature.

$$
P_{\mathrm{cool}}=I\left(\frac{\hbar \omega}{q} \eta_{\mathrm{EQE}}\right)-I^{2} R .
$$

Note that $R$ is not indicative of a purely irreversible process as in an Ohmic resistance. At low bias, voltage and current are directly proportional and $R$, measured in ohms, represents their ratio.

Equation (2) indicates that net cooling results from competition between a cooling process linear in current and a heating process quadratic in current. Here, low-bias LED operation is analogous to a thermoelectric cooler [24], in which Peltier heat transfer competes with Joule heating to realize heat pumping. In both devices a finite current maximizes cooling power, and at lower currents there is a trade-off between power and efficiency. Moreover, as sources of irreversibility are removed from the LED, it acts as a reversible Carnot-efficient heat pump $[19,25]$ operating between the lattice and the photon field.

Figures 4(a) and 4(b) show that both the maximum output power at unity efficiency and the maximum cooling power increase with temperature.

When a small voltage is applied, the energy barrier to thermally assisted injection is lowered so that the product $n p$ rises as in the Shockley diode equation [26]:

$$
n p=n_{0} p_{0} e^{q V / k_{\mathrm{B}} T} .
$$

Hence, for a given magnitude of the dimensionless excitation $q V / k_{B} T$, net recombination is proportional to the product of equilibrium electron and hole concentrations $n_{0} p_{0}$. For nondegenerate regions, this reduces to the intrinsic carrier concentration squared. Since this quantity is a measure of the ambient environment's ability to thermally excite electrons across the band gap, it is exponentially dependent on the ratio $E_{\text {gap }} / k_{B} T$ [25]:

$$
n_{0} p_{0} \propto e^{-E_{\text {gap }} / k_{B} T} .
$$

This largely explains the low-bias temperature dependences in Figs. 3 and 4, and moreover why cooling in the low-bias regime is not considered for the GaAs $[4,6]$ and InP [7] designs put forth in recent years. Equation (4) suggests that switching from $135^{\circ} \mathrm{C}$ InGaAsSb (where $E_{\text {gap }} / k_{B} T \approx 15$ ) to these wider band gap materials at $25^{\circ} \mathrm{C}$ (where $E_{\text {gap }} / k_{B} T>50$ ) would result in a reduction of the optical power available at low bias by roughly 15 orders of magnitude.

Shown alongside experimental data in the figures are the results of a one-dimensional simulation [21] of the first three moments of the Boltzmann transport equation, a lattice heat diffusion equation, and an optical transmission calculation. The carrier transport equations were solved self-consistently with the lattice heat diffusion equation using a commercial software package. The recombination mechanisms in the carrier transport model were bulk trapbased SRH, surface SRH at the heterointerfaces, radiative bimolecular, Auger, and surface recombination at the contacts. The optical transmission calculation was used to correct for the effects of photon recycling and changes in extraction at high bias. The simulation included three fitting parameters: a series contact resistance of $0.779 \Omega$, a nonradiative bulk SRH lifetime at $300 \mathrm{~K}$ of $95 \mathrm{~ns}$, and a collection efficiency of $24.5 \%$ used only in the simulation. This collection efficiency indicates that the experimentally measured $231 \%$ wall-plug efficiency corresponds to a simulated internal wall-plug efficiency of $943 \%$.

In addition to the intrinsic value of experimental confirmation of electroluminescent cooling, several potential applications present themselves. Although the heat pumping power density of the particular device examined here was too low to provide practical cooling at room temperature, the result demonstrates the relevance of $\eta_{\mathrm{EQE}}$ below typical operating voltages for light emitters designed for lighting without heat generation [3,4,6], solid-state cooling [3-10,17,27], or power generation [28]. The importance of this quantity has been theoretically explored by Heikkila, et al. [3] and is captured in Eq. (1). 
Furthermore, the extremely efficient electroluminescence available at subthermal voltages could enable efficient high-temperature sources for lock-in spectroscopy. The device examined here could serve as an efficient source around $4130 \mathrm{~cm}^{-1}$ in a $135^{\circ} \mathrm{C}$ environment. As the wavelength is extended toward longer wavelengths, the associated decrease in $E_{\text {gap }} / k_{B} T$ should improve LED output power and widen the temperature range over which high efficiency is attained. In still highertemperature environments, low-bias operation of infrared LEDs may be much more efficient and diminish the need for any active cooling [29]. For example, both the analysis of exhaust gases from combustion and down-hole spectroscopy rely on infrared sources operating at elevated temperature.

We also note that the LED in this experiment responds to electrical excitation with photon emission on time scales faster than typical thermal time constants for solids of a similar dimension. As a result, the arbitrarily efficient generation of photons at low bias may offer a new platform to test the limits of energy-efficient electromagnetic communication.

Finally, the results reported here were measured on a device designed for conventional high-bias operation. Designing LEDs for low-bias operation, for example, by doping the active region $[3,30]$, should raise low-bias $\eta_{\mathrm{EQE}}$ and therefore the optical power density available with no net heat generation as well. Moreover, further reduction of $E_{\text {gap }} / k_{B} T$ should produce further increases in power density. Where elevated emitter temperatures are necessary, including in shorter wavelength sources, self-heating may offer a convenient solution for sources with subunity $\eta$. Here, purposeful concentration of internally generated heat, such as in an incandescent filament, should allow phonons to be recycled to thermally pump the emitter.

In conclusion, a new regime for electroluminescent cooling of a semiconductor diode was experimentally demonstrated. In this regime application of a forward bias voltage $V$ less than the thermal voltage $k_{B} T / q$ imposes a small deviation from thermodynamic equilibrium on the device. In response, the rates of both radiative and nonradiative recombination in the device's active region have contributions at linear order in $V$, so that the external quantum efficiency $\eta_{\mathrm{EQE}}$ is voltage independent. As a result, the LED's optical output power scales linearly with voltage while the input power scales quadratically, resulting in arbitrarily efficient photon generation accompanied by net electroluminescent cooling of the solid at low bias. Experimental evidence was presented for small band gap emitters at high temperature.

Supported in part by the NDSEG fellowship program. The authors thank B. Matveev for sharing details about the LED21Sr.
*To whom correspondence should be addressed. rajeev@mit.edu

[1] J. Tauc, Czech. J. Phys. 7, 275 (1957).

[2] A. Weinstein, J. Opt. Soc. Am. 50, 597 (1960).

[3] O. Heikkila, J. Oksanen, and J. Tulkki, J. Appl. Phys. 105, 093119 (2009).

[4] O. Heikkila, J. Oksanen, and J. Tulkki, J. Appl. Phys. 107, 033105 (2010).

[5] S.-Q. Yu et al., Proc. SPIE Int. Soc. Opt. Eng. 6486, 648604 (2007).

[6] S.-T. Yen and K.-C. Lee, J. Appl. Phys. 107, 054513 (2010).

[7] P. Han, K.-J. Jin, Y.-L. Zhou, H.-B. Lu, and G.-Z. Yang, J. Appl. Phys. 101, 014506 (2007).

[8] A. G. Mal'shukov and K. A. Chao, Phys. Rev. Lett. 86, 5570 (2001)

[9] J.-B. Wang, D. Ding, S.-Q. Yu, S. R. Johnson, and Y.-H Zhang, in Proceedings of the Quantum Electronics and Laser Science Conference, Baltimore, MD, 2005 (Optical Society of America, Washington, DC, 2005), Vol. 1, pp. 655-657, paper QThI7.

[10] J. Oksanen and J. Tulkki, Proc. SPIE Int. Soc. Opt. Eng. 7951, 79510H (2011).

[11] K. P. Pipe, R. J. Ram, and A. Shakouri, Phys. Rev. B 66, 125316 (2002).

[12] K. Lehovec, C. A. Accardo, and E. Jamgochian, Phys. Rev. 89, 20 (1953).

[13] R. Newman, Phys. Rev. 91, 1313 (1953).

[14] J. R. Haynes and W.C. Westphal, Phys. Rev. 101, 1676 (1956).

[15] P. T. Landsberg and D. A. Evans, Phys. Rev. 166, 242 (1968).

[16] P. T. Landsberg and G. Tonge, J. Appl. Phys. 51, R1 (1980).

[17] W. Nakwaski, Electron Technol. 13, 61 (1982).

[18] G. C. Dousmanis, C. W. Mueller, H. Nelson, and K. G. Petzinger, Phys. Rev. 133, A316 (1964).

[19] P. Berdahl, J. Appl. Phys. 58, 1369 (1985).

[20] Data Sheet for Ioffe LED, Ltd. Model LED21Sr.

[21] See Supplemental Material at http://link.aps.org/ supplemental/10.1103/PhysRevLett.108.097403 for details regarding the experimental methods and the numerical model.

[22] W. Shockley and W. T. Read, Phys. Rev. 87, 835 (1952).

[23] R. N. Hall, Phys. Rev. 87, 387 (1952).

[24] G.S. Nolas, J. Sharp, and H.J. Goldsmid, Thermoelectrics: Basic Principles and New Materials Developments (Springer-Verlag, Berlin, 2001).

[25] C. Kittel and H. Kroemer, Thermal Physics (W. H. Freeman and Co., New York, 1980), 2nd ed.

[26] E. F. Schubert, Light-Emitting Diodes (Cambridge University Press, Cambridge, England, 2003).

[27] W. Bradley, U.S. Patent No. 2,898,743 (1959).

[28] N.-P. Harder and M. A. Green, Semicond. Sci. Technol. 18, S270 (2003).

[29] V. Malyutenko, A. Melnik, and O. Malyutenko, Infrared Phys. Technol. 41, 373 (2000).

[30] D. Ban et al., J. Appl. Phys. 96, 5243 (2004). 